AperTO - Archivio Istituzionale Open Access dell'Università di Torino

\title{
Pharmacological treatments and risks for the food chain
}

\section{This is the author's manuscript}

Original Citation:

Availability:

This version is available http://hdl.handle.net/2318/45681

since

Published version:

DOI:10.1107/s11259-008-9083-5

Terms of use:

Open Access

Anyone can freely access the full text of works made available as "Open Access". Works made available under a Creative Commons license can be used according to the terms and conditions of said license. Use of all other works requires consent of the right holder (author or publisher) if not exempted from copyright protection by the applicable law. 


\title{
Pharmacological treatments and risks for the food chain
}

\author{
C. Girardi • R. Odore
}

Published online: 7 August 2008

(C) Springer Science + Business Media B.V. 2008

\begin{abstract}
Veterinarians play a pivotal role in public health control, in particular in the management of risks deriving from pharmacological treatments of food-producing animals. Veterinary medicinal products can represent a risk for animal health and welfare (side effects, decreased efficacy), for farmers and practitioners administering the drug, for consumers of food of animal origin (presence of residues, occurrence of antibiotic resistance) and for the environment. According to pending European guidelines, risk management starts from marketing authorisation that must be based on risk evaluation and can be denied when the risk/benefit ratio is not favourable considering the advantages for animal health and welfare and for safety of consumers. Veterinarians can prevent and control risks by using correct pharmacological criteria to choose and administer medicinal products and undertaking risk-based inspection of residues of drugs in food of animal origin. Moreover, a major tool for veterinarians to prevent and control drug-borne risk is "pharmacovigilance". Risks for the environment are usually assessed during the premarketing approval process, however veterinarians, as risk managers, should educate farmers about correct drug handling and disposal, and periodically verify that suggested measures are applied.
\end{abstract}

Keywords Food chain $\cdot$ Pharmacovigilance $\cdot$ Residue $\cdot$ Risk $\cdot$ Veterinary drug

\section{Introduction}

The role of veterinarians in public health control is crucial at various points along the food chain, starting from the preservation of animal health to the inspection process for food of animal origin (Mumford and Kihm 2006). As the concept of "zero tolerance level" is no longer acceptable in

R. Odore

Department of Animal Pathology, Division of Veterinary Pharmacology and Toxicology,

University of Turin, Turin, Italy

C. Girardi $(\bowtie)$

Dipartimento di Patologia Animale, Facoltà di Medicina Veterinaria, via Leonardo da Vinci 44, 10095 Grugliasco, (TO), Italy

e-mail: carlo.girardi@unito.it 
a modern production system, it has become important to identify a risk-based approach at both national and international level and to adopt standard risk analysis processes.

The use of veterinary drugs can represent a risk for animal health and animal welfare, for farmers or veterinarians administering the drug and for consumers of food products of animal origin. Moreover, it should be remembered that veterinary drugs, shed by the treated animals with their excretions, can represent a risk for various environmental media, including soil, surface and groundwater, suggesting the potential for indirect human exposure from such residues (Capleton et al. 2006).

Establishment of a legislative framework and of an institutional structure is the first step in the assessment and management of drug-related risk. From this point of view, according to pending European legislation the use of veterinary drugs must be based on risk evaluation. The risk due to the use of veterinary drugs is "any risk for animal or public health relating to the quality, safety and efficacy of the veterinary medicinal product and any risk of undesirable effect on the environment".

Risk management is a task of both private and public veterinary services that are involved in the prevention and control of all hazards arising from the use of veterinary drugs. A major tool for veterinarians to prevent and control drug-borne risk is "pharmacovigilance".

\section{Risks for treated animals and for people administering drugs}

Despite the fact that application for marketing authorisation of a veterinary medicinal product is based on pharmacological and toxicological studies, data are generally obtained from a limited number of animals, whereas extensive use of the drug can cause adverse effects in some animal breed or category or during specific diseases.

Pharmacovigilance is the post-marketing surveillance of veterinary drug and vaccine safety used for prevention, diagnosis and therapy and consists of the report of any adverse effects of a drug by veterinarians, pharmacists, farmers and other health care professionals, in the improvement of knowledge about the pharmacological action of a drug and hence, in the evaluation of the risk/benefit balance of a drug (Keck and Ibrahim 2001). Despite the fact that the reporting of adverse drug effects has been advocated for a long time, the general apathy in fulfilling this duty has prompted national and international authorities to make reporting a legal obligation. This has led to the birth of a new discipline in Veterinary Medicine, pharmacovigilance, regulated at a European level by Directive 2004/28/CE. The main tasks of pharmacovigilance can be summarized as follows:

- control of clinical safety of veterinary medicinal products;

- control of potential reaction in man linked to user safety;

- evaluation of decreased efficacy or lack of expected activity of a veterinary medicinal product;

- control of maximum residue levels (MRL) of veterinary drugs in food products of animal origin;

- assessment of risks for the environment related to the use of veterinary drugs;

- control of the development of drug resistance, with particular concern to antibiotic resistance.

It appears clear that, in veterinary medicine, pharmacovigilance should provide useful information related not only to animal health and welfare, but also to consumers of food products of animal origin. Good pharmacovigilance practice is the responsibility of everyone involved in the development, authorisation, marketing and use of veterinary 
drugs. In this regard, veterinarians play a pivotal role and their report concerning adverse drug effects represents an important strategy of risk management.

Veterinarians must promptly report to the competent authorities any suspected adverse drug reaction, particularly serious adverse reactions (i.e. life-threatening effects or reactions that lead to congenital defects, permanent or prolonged clinical signs, significant disability or incapacity or death in treated animals). The analysis of reports collected by a well organized pharmacovigilance centre can lead to product safety recommendations, change in labelling, modification of drug use or, in rare circumstances, removal of the product or even withdrawal of approval from the market. As mentioned before, one of the aims of pharmacovigilance is the surveillance of the potential adverse effects by veterinary drug handlers. An example is represented by the reports of accidental human exposures to tilmicosin. The observation of serious adverse cardiovascular effects in humans accidentally exposed to needle puncture of medicinal products containing the antibiotic has led to special care in drug handling; as a result the administration of tilmicosin is now restricted to use by veterinarians only (Veenhuizen et al. 2006).

\section{Potential risks of drug residues in food products of animal origin}

Human health risk can result from the presence of residues of veterinary drugs and/or their metabolites in edible organs and tissues of treated animals, in particular residues in concentrations exceeding the MRL established by Council Regulation 2377/90.

Potential adverse effects from residues include not only acute reactions such as the aplastic anemia caused by chloramphenicol, allergic reactions induced by beta-lactam antibiotics and adrenergic symptoms due to $\beta_{2}$-agonists, but also chronic effects, for example immunodepression, teratogenicity, mutagenicity and carcinogenicity (Gehring et al. 2006). It is worth mentioning that the occurrence of collective poisoning following consumption of contaminated food products has only been described for clenbuterol (Barbosa et al. 2005). The lack of epidemiological data may be due to the inadequacy of reporting, the absence of definitive diagnosis of alimentary poisoning, the difficulty in finding the contaminated food product, or to the fact that, at present, many of the potential risks linked to the presence of drug residues or metabolites have not been elucidated. Furthermore, residues can generally be found at very low concentrations and are more likely implicated in the occurrence of sub-acute or chronic effects rather than acute reactions.

A particular concern is the slow tissue clearance of residues of some injectable formulations from the site of administration. The primary issue is consumer safety in view of the potential acute consequences resulting from the ingestion of injection site residues. Licensing authorities have adopted different approaches to the human food safety assessment of injection site residues (Reeves 2007). European agencies apply the maximum residue limit for muscle tissue to the muscle injection site and specify a withdrawal period sufficient to ensure that ingestion of a $300 \mathrm{~g}$ piece of muscle, even if it were comprised entirely of injection site tissue will not exceed the acceptable daily intake (ADI). For drugs in Annex II of Council Regulation 2377/90, for which MRLs have not been determined, the safety parameter to be considered is the ADI. The Australian, Canadian and American food inspection agencies also exclude injection site residues from the MRL-setting process. These authorities evaluate the risk for consumers to develop potential acute manifestations after infrequent ingestion of injection site residues based on acute dietary exposure estimates. In this case, the ADI is substituted by the ARfD (Acute Reference Dose), the 
total dose of residues of a chemical in the food that can be ingested over a short period, usually one meal or one day, without appreciable health risk to the consumer (Reeves 2007).

Besides the fact that a concerted approach to risk analysis is needed, on-farm measures to mitigate the risks associated with injection site lesions can be undertaken. The persistence of residues at injection sites is commonly observed with irritant drugs and may be associated with tissue fibrosis and necrosis causing the drug to be incapsulated in injection site tissue. It is therefore important that licensing Authorities review tissue irritancy data for all formulations intended for intramuscular administration during the pre-marketing drug approval process. Moreover, maximum volume administered per injection site should be limited and drugs should be injected in specific anatomical regions such as in the neck (George et al. 1997). In the US, the incidence of injection site lesions in beef rumps has decreased from $21 \%$ in 1991 to $2.1 \%$ in 2000 as a result of quality assurance programme implementation by the livestock industry (Roeber et al. 2001).

For veterinarians, the management of risks arising from the presence of residues in food products starts with a careful prescription and administration of drugs with low MRL values (i.e. corticosteroids) and involves risk-based food inspection.

An important aspect of risk management is the control and prevention of all the conditions that may potentially constitute a risk. In particular, veterinarians responsible for drugs stocked at the farm should avoid a series of off-label uses of medicinal products. It should be noted that as far as drugs intended for food-producing animals are concerned, according to pending legislation, the extralabel use is restricted, by way of derogation, to:

1. use of an approved drug in a different animal species or for the treatment of a different condition in the same species;

2. use of a drug approved for humans;

3. use of a drug authorised in a different Member State;

4. use of a drug prepared extemporaneously by a pharmacist with the term of a veterinary prescription.

In all these cases, a prescribing cascade has been established to guide veterinarians in their decision-making process. Veterinarians cannot prescribe a drug unless the active ingredients appear in Annexes I, II or III of Council Regulation 2377/90 and should indicate precautionary withdrawal periods to avoid the presence of harmful residues in edible tissues. Any other off-label use of veterinary drugs is prohibited, thus illegal. Veterinarians have to inform farmers about potential risks and legal consequences arising from uses such as:

1. an alternative, non-authorised, route of administration;

2. administration of the drug in a different animal species or category of animals, for example the administration of ivermectin in animals from which milk is produced for human consumption or of fluoroquinolones in animals producing eggs for human consumption;

3. administration of drugs containing active ingredients that cannot be used in foodproducing animals and are therefore included in Annex IV of Council Regulation 2377/ 90 (i.e. chloramphenicol or nitrofuranes);

4. administration of approved drugs for different purposes or in non-authorised species as in the case of steroids administered as anabolic agents and of $\beta_{2}$-adrenergic drugs used as repartitioning agents. It has been well documented that these illegal treatments, 
besides representing a risk for consumers, induce anatomical, biochemical and endocrine modifications in treated animals and seriously threaten their well being;

5. administration of an approved drug altering the dose, particularly for antimicrobial drugs and antiparasitic drugs. While increasing the dose could potentially result in the need for extended withdrawal times, sub-therapeutic dosages could promote drug resistance.

\section{Antibiotic resistance}

The problem of antibiotic resistance represents a challenge for veterinarians involved in the management of drug-related risks. The development of resistance can be minimised provided that a number of measures are observed to prolong the useful life of all antibiotics both in human and veterinary medicine. The scientific knowledge provides the basis for judicious therapeutic antimicrobial use by veterinarians (Passantino 2007). Since the early 1960 s, the risk of antibiotic resistance due to the use of antimicrobial drugs in foodproducing animals has been known. In some circumstances, the phenomenon of resistance has been associated with the continuous administration of sub-therapeutic doses of antimicrobials, as in the case of performance enhancers. In countries where avoparcin (a glycopeptide antibiotic, like vancomycin) is or has been used as a growth promoter, vancomycin resistance is common in intestinal enterococci not only in exposed animals but also in the human population outside the hospitals (van den Bogaard and Stobberingh 2000). Resistance genes against antibiotics used in animals have been found soon after their introduction, not only in animal bacteria or in the commensal flora of humans, but also in zoonotic pathogens like salmonellae and in strictly human pathogens like shigellae. This demonstrates the transfer of resistance genes between human and animal bacteria. In Sweden and the US, where avoparcin has never been used, no high level vancomycin resistant enterococci have been found in faecal samples of food-producing animals or healthy animals. The concern about promotion and spread of resistance has led to the ban of antibiotics as growth promoters. Moreover, since January 1st 2006, the EU banned certain antimicrobials used in food-producing animals as feed supplements.

Despite the fact that the use of antimicrobials as growth promoters is often linked to resistance, one cannot exclude that other drugs used for therapeutic purposes can elicit similar risks. The rapid increase in the prevalence of fluoroquinolone resistance among human Campylobacter isolates was a cause of concern during the late 1990s (Nelson et al. 2007). It was shown that in flocks infected with Campylobacter jejuni, the selection of resistant isolates emerged after therapy with enrofloxacin (Jacobs-Reitsma et al. 1994). In Great Britain, enrofloxacin was registered for veterinary use in 1993 and in that year only $1 \%$ of locally raised poultry was fluoroquinolone resistant. In 1997 the percentage had approached a level of about $10 \%$. This led to the ban of fluoroquinolones in poultry producing eggs for human consumption (Nelson et al. 2007).

It is difficult to estimate the actual risk of drug resistance following treatment of foodproducing animals. There are several other sources of the problem, including inappropriate use of antibiotics in humans. Nevertheless, the isolation of multidrug resistant micro organisms in human infections suggest prudent use of this category of drugs in food animals. Maintaining a low level or resistance in the intestinal flora of food animals should be one of the main goals of modern breeding systems and safe food trades.

Veterinarians have to adopt strategies that help prevent and control the bacterial infection, therefore limiting the use of antibiotics and/or using rationale criteria to choose therapeutic protocols in light of a favourable risk/benefit/cost ratio (Baker 2006). In 
Denmark the total ban of antibiotics as growth promoters has reduced the use of these drugs from 208 tons in 1994 to 81 tons in 1999. Furthermore, strategies to prevent and control the spread of infectious diseases (visits to the farm every month, prescription based on actual prevalence of disease, prescribed medicines delivered by pharmacy, written instructions for drug use, written description of actions taken to avoid disease, reports of visits, and daily and monthly recording of drugs used by farm staff) have demonstrated that appropriate diet and better farm management at all levels are important factors that can compensate for the lost effects of growth enhancing antibiotics (Jensen 2006).

The choice of the right antibiotic should be based on microbiological and pharmacological criteria; applying the pharmacokinetic/pharmacodynamic (PK/PD) properties allows, in fact, prediction of antimicrobial efficacy (Passantino 2007). According to GVP (Good Veterinary Practices) guidelines, the microbiological diagnosis is always necessary and the most suitable antibiotic should be chosen based on the following criteria:

- $\quad$ spectrum of activity as narrow as possible;

- good tissue penetration;

- $\quad$ margin of safety as high as possible;

- use authorised in veterinary medicine.

Antimicrobials used as a last resort in human medicine (fluoroquinolones and last generation cephalosporins) should only be used in individual animals and in a limited number of cases only or under strict indications where other antibiotics fail (Ungemach et al. 2006).

In the European Union there are two main directives functioning as effective instruments for managing risks related to antimicrobial resistance: a guideline on pre-authorisation designed to assess the potential for resistance development from the use of an antimicrobial veterinary product (EMEA/CVMP/244/01) and a guideline governing the general veterinary use of antimicrobials in target animal species (EMEA/CVMP/627/01) (Grugel and Wallmann 2004).

\section{Veterinary drugs and environmental risks}

A number of studies has documented the presence of drugs and/or drug metabolites in the environment: the problem has received a certain degree of attention when measurable concentrations of cocaine and other illicit drugs were found in affluents and effluents of some Italian wastewater treatment plants (Castiglioni et al. 2006).

As far as veterinary drugs are concerned, many active ingredients have been detected in various environmental media, especially drugs used for herd treatments. In Germany and the US, for example, low levels $(<1 \mu \mathrm{g} / \mathrm{l})$ of antibiotic residues have been found in surface water samples taken from sites considered susceptible to contamination (Hirsch et al. 1999).

Veterinary drugs can enter the environment in a number of different ways:

- $\quad$ direct application as in aquaculture systems;

- with the excretions of treated animals;

- via application of manure or slurry to farmland;

- during the manufacturing and formulation processes;

- through disposal.

The fate and behaviour of active ingredients in the environment mainly depends on their physico-chemical characteristics, but also on the nature and properties of the soils and water 
in which the drug is eliminated. Tetracycline, for example, tends to adhere strongly to soils and, as a consequence, cannot be detected in surface and groundwater samples. By contrast, sulfonamides are rapidly transported to surface waters, the process being dependent on the soil and field type: in fact the transport of sulfonamides is limited in the presence of sandy soil and manure arable plots. Moreover, accumulation in the environment depends also on the rate of degradation in the different media: in cattle, poultry and swine slurry tylosin degrades much faster than chlortetracycline, tilmicosin and lincomycin (Zilles et al. 2005).

It is quite difficult to exactly estimate the risk of human exposure to drugs present in the environment. However, it is possible to use some criteria to assess the potential of active ingredients of veterinary drugs to reach the environment, their usage and their toxicity profiles in order to predict the likelihood that indirect exposure of the general population might occur (Capleton et al. 2006). The potential to reach the environment depends on the target treatment group (companion animals, aquaculture, herd animals), the route of administration (drugs administered topically are considered at higher risk) and the metabolic rate in the treated animal (higher risk for drugs with low metabolism i.e. less than 20\%). Usage can be categorized on the basis of chemical groups: undoubtedly drugs at high usage (>tonnes per annum) have a higher potential for indirect exposure than those used in small amounts $(<1$ tonne per annum). The toxicity profile can be estimated by defining the ADI, the potential for bioaccumulation and the presence of biologically active metabolites. On the basis of the previously mentioned criteria, some drugs such as betalactam antibiotics and some antiparasitic agents (albendazole, levamisole) can be classified as "high" priority for detailed risk assessment (Capleton et al. 2006).

Recently, the development of in-vitro antimicrobial resistance in bacteria exposed to residual concentrations of some antibiotics ( $\mathrm{ppb}$ ) has been studied. The increased level of resistance suggests that even levels of antibiotics similar to those found in the environment can have a biological activity, representing a risk for the selection of resistant bacteria (Kleiner et al. 2007).

The application for registration of veterinary drug products for food-producing animals requires that a risk assessment for the environment is submitted allowing the licensing authority or agency to deny approval in case of a serious environmental risk. Special precautions for disposal of high risk veterinary drugs and excretion of treated animals should be taken. Despite the fact that specific recommendations are generally suggested in the package insert, veterinarians, as risk managers, should educate farmers about correct drug handling and disposal, and periodically verify that suggested measures have been applied.

\section{References}

Baker R., 2006. Health management with reduced antibiotic use. The US experience. Animal Biotechnology, 17, 195-205.

Barbosa J., Cruz C., Martins J., Silva J.M., Neves C., Alves C., Ramos F., Da Silveira M.I., 2005. Food poisoning by clenbuterol in Portugal. Food Additives and Contaminants, 22, 563-566.

Capleton A.C., Courage C., Rumsby P., Holmes P., Stutt E., Boxall A.B.A., Levy L.S., 2006. Prioritising veterinary medicines according to their potential indirect human exposure and toxicity profile. Toxicology Letters, 163, 213-223.

Castiglioni S., Zuccato E., Crisci E., Chiabrando C., Fanelli R., Bagnati L., 2006. Identification and measurement of illicit drugs and their metabolites in urban wastewater by liquid cromatography-tandem mass spectrometry. Analytical Chemistry, 78, 8421-8429.

Gehring R., Baynes R.E., Riviere J.E., 2006. Application of risk assessment and management principles to the extralabel use of drugs in food-producing animals. Journal of Veterinary Pharmacology and Therapeutics, 29, 5-14. 
George M.H., Cowman G.L., Tatum J.D., Smith G.C., 1997. Injection-site lesions in beef subprimals: incidence, palatability consequences, and economic impact. Compendium of Continuing Education for Practising Veterinarians, 19, S84-S93.

Grugel G. and Wallmann J., 2004. Antimicrobial resistance in bacteria from food-producing animals, risk management tools and strategies. Journal of Veterinary Medicine B, 51, 419-421.

Hirsch R., Ternes T., Haberer K., Kratz K.L., 1999. Occurrence of antibiotics in the aquatic environment. The Science of Total Environment, 225, 109-118.

Jacobs-Reitsma W.F., Kan C.A., Bolder N.M., 1994. The induction of quinolone resistance in Campylobacter bacteria in broilers by quinolone treatment. Letters in Applied Microbiology, 19, 228231.

Jensen H.M., 2006. Health management with reduced antibiotic use. Experiences of a Danish pig Vet. Animal Biotechnology, 17, 189-194.

Keck G. and Ibrahim C., 2001. Veterinary pharmacovigilance: between regulation and science. Journal of Veterinary Pharmacology and Therapeutics, 24, 369-373.

Kleiner D.K., Stanley E.K., Ward P.L.M., 2007. Development of in vitro antimicrobial resistance in bacteria exposed to residual level exposures of antimicrobial drugs, pesticides and veterinary drugs. Chemotherapy, 53, 132-136.

Mumford E.L. and Kihm U., 2006. Integrated risk reduction along the food chain. Annals of the New York Academy of Sciences, 1081, 147-152.

Nelson J.M., Chiller T.M., Powers J.H., Angulo F.J., 2007. Fluoroquinolone resistant Campylobacter species and the withdrawal of fluoroquinolones from use in poultry: a public health success story. Food Safety, 44, 977-980.

Passantino A., 2007. Ethical aspects for veterinarians regarding antimicrobial drug use in Italy. International Journal of Antimicrobial Agents, 29, 240-244.

Reeves P.T., 2007. Residues of veterinary drugs at injection sites. Journal of Veterinary Pharmacology and Therapeutics, 30, 1-17.

Roeber D.L., Cannell R.C., Belk K.E., Scanga J.A., Cowman G.L., Smith G.C., 2001. Incidence on injection site lesions in beef top sirloin butts. Journal of Animal Science, 79, 2615-2618.

Ungemach F., Muller-Bahrdt D., Abraham G., 2006. Guidelines for prudent use of antimicrobials and their implications on antibiotic usage in veterinary medicine. International Journal of Medical Microbiology, 296, 33-38.

van den Bogaard A.E. and Stobberingh E.E., 2000. Epidemiology of resistance to antibiotics. International Journal of Antimicrobial Agents, 14, 327-335.

Veenhuizen M.F., Wright T.J., McManus R.F., Owens J.G., 2006. Analysis of reports of human exposure to Micotil 300 (tilmicosin injection). Journal of Veterinary Medical Association, 229, 1737-1742.

Zilles J., Shimada T., Jindai A., Robert M., Raskin L., 2005. Presence of macrolide-lincosamidestreptogramin B and tetracycline antimicrobials in swine waste treatment process and amended soil. Water Environment Research, 77, 57-62. 